\title{
Scaling at the Chaos Threshold for Interacting Electrons in a Quantum Dot
}

\author{
$\mathrm{X}$ Leyionas, ${ }^{1}{ }^{1}$ P G Silvestrov, ${ }^{12}$ and $\mathrm{C}$ W J Beenakke1 ${ }^{1}$ \\ ${ }^{1}$ Instituut Lorentz Unversitelt Leiden PO Box 95062300 RA Leiden The Netherlands \\ ${ }^{2}$ Budker Institute of Nucleal Ph)sics 630090 Novosibirsk Russia
}

(Recelved 29 November 1999)

\begin{abstract}
The chaotic mixing by 1 andom two body interactions of many-electron Fock states in a confined geometiy is investigated Two regimes are distinguished in the dependence of the typical number of Fock states that are mixed into an eigenstate on the interaction stiength $V$, the excitation energy $\varepsilon$, and the level spacing $\Delta$ In both regimes the number is latge (indicatıng delocalization in Fock space) Howevel, only the laige- $V$ regime is descubed by the golden rule (indicating chaotic mixing) The clossover region is characterized by a maximum in a scaling function that becomes mote pronounced with increasing excitation energy The scaling parameter that governs the transition is $\left(\varepsilon V / \Delta^{2}\right) \ln (\Delta / V)$
\end{abstract}

PACS numbers $7323-\mathrm{b} \quad 0545-\mathrm{a} 7110-\mathrm{w}$

The highly excited atomic nucleus was the first example of a quantum chaotic system, although the interpietation of Wignei's distribution of level spacings [1] as a signatuie of quantum chaos came many years later, fiom the study of election billiards [2] While the spectial statistics of the nucleus and the billiaid are basically the same, the origin of the chaotic behavior is entırely diffeient [3] In the billıard chaos appears in the single particle specti um as a result of boundary scattering, while in the nucleus chaos appears in the many-particle spectrum as a result of interactions

The study of the interaction-induced tiansition to chaos entered condensed matter physics with the realization that a semiconductor quantum dot could be seen as an attificial atom or compound nucleus [4] A particularly influential paper by Altshuler, Gefen, Kamenev, and Levitov [5] studied the interaction-induced decay of a quasiparticle in a quantum dot and interpreted the broadening of the peaks in the single-particle density of states as a delocalization transition in Fock space Different scenaios leading to a smooth rather than an abrupt transition fiom localized to extended states were considered later [6-8] Recent computer simulations $[9,10]$ also confirm the smooth ciossover fiom localızed to delocalızed regime for quasıpatıcle decay

As emphasized by Altshuler et al [5], the delocalized regime in the quasiparticle decay problem is not yet chaotic because the states do not extend uniformly over the Fock space One may study the transition to chaos in the singleparticle density of states, but theoretically it is easier to consider instead the mixing by interactions of arbitrary many-particle states This was the approach taken in Refs $[6,8,11-14]$, focusing on two quantities The distribution of the energy level spacings and the inverse paticipation iatio (IPR) of the wave functions in Fock space Both quantities can serve as a signature for chaotic behavior, the spacing distribution by comparing with Wignei's distribution [1] and the IPR by compaing with the golden iule (according to which the IPR is the mean spacing $\delta$ of the many-particle states divided by the mean decay rate $\Gamma$ of a noninteractung many-patticle state [12]) Two fun- damental questions in these investigations are as follows (1) What is the scaling parameter that governs the transi tion to chaos? (2) How shaip is the tiansition?

In a tecent paper [14] one of us presented analytical at guments for a singular threshold governed by the scaling parameter $x=(\varepsilon / g \Delta) \ln g$, where $\Delta$ is the single particle level spacing, $\varepsilon$ is the excitation energy, and $g$ is the con ductance in units of $e^{2} / h$ (Both $\varepsilon / \Delta$ and $g$ are assumed to be $\gg 1$ ) In contıast, Georgeot and Shepelyansky [12] argued for a smooth crossover governed by the parame te1 $y=(\varepsilon / g \Delta) \sqrt{\varepsilon / \Delta}$ (The same scalıng parameter was used in Refs $[6,13])$ The parameter $y$ is the ratio of the stiength $V \sim \Delta / g$ of the scieened Coulomb interaction $[5,15]$ and the eneigy spacing $\Delta_{2} \sim(\varepsilon / \Delta)^{-3 / 2} \Delta$ of states that are directly coupled by the two-body interaction [6] The parameter $x$ follows if one considers contributions to the IPR that involve the effective interaction of $2,3,4$, , particles Subsequent terms in this series are smalle1 by a factor (lng/g) $\Delta_{n} / \Delta_{n+1}$, where $\Delta_{n} \sim(\varepsilon / \Delta)^{-n+1 / 2} \Delta$ is the spacing of states that are coupled by an effective interactıon of $n$ partıcles [14] (The large logar thm $\ln g$ appears in the expansion parameter because of the large contribu tion fiom intermediate states whose eneigies are close to the states to be mixed )

The purpose of this paper is to investigate the interaction-induced transition to chaos by exact diagonalization of a model Hamiltonian We concentrate on the IPR because for that quantity an analytical prediction exists [14] for the $\varepsilon$ and $g$ dependence (There is no such piediction for the spacing distribution) The numerical data are consistent with a chaos threshold at a value of $x$ of otder unity Our model is the same as that used by Georgeot and Shepelyansky [12] The difference in scalıng parameter with Ref [12] may be due in part to the fact that no analytical theory to compare with was avarlable at that time, and in part to the fact that most of the numerics in that paper was done for nondegenerate systems (number of accessible single-particle states much gieater than the number of particles) - Instead of the highly degenerate system considered here 
The model for interacting spinless fermions that we study is the layer model introduced in Ref [12] and used for the quasiparticle decay problem in Ref [10] The Hamiltonian is $H=H_{0}+H_{1}$, with

$$
H_{0}=\sum_{l} \varepsilon_{l} c_{l}^{\dagger} c_{l}, \quad H_{1}=\sum_{l<J k<l} V_{l j k l} c_{l}^{\dagger} c_{k}^{\dagger} c_{l} c_{J}
$$

The single-particle levels $\varepsilon$, are uniformly distributed in the inter val $\left[\left(j-\frac{1}{2}\right) \Delta,\left(J+\frac{1}{2}\right) \Delta\right]$ The interaction matıx elements $V_{l, k l}$ are zero unless $t, j, k, l$ are four distinct indices with $l+j=k+l$ The (real) nonzero matilx elements have a Gaussian distribution with zero mean and var rance $V^{2}=(\Delta / g)^{2}$ (This relationship between interaction stiength and dimensionless conductance for a dif fusive quantum dot has been derived in Refs [5,15]) The Fock states are elgenstates of $H_{0}$, given by Slater dete1minants of the occupred levels $k_{1}, k_{2}, k_{3}$, The interaction mixes Fock states for which $\sum_{p} k_{p}$ equals a given integer (Without this restriction the model is the same as the two-body iandom-interaction model intioduced in nuclear physics $[16,17])$ The excitation energies of the states with given $k_{1}, k_{2}, k_{3}$, , lie in a relatively narrow layel (w1dth of ordet $j^{1 / 4} \Delta$ ) around the mean excitation energy $J \Delta$ The number of states in the $j$ th laye is the number of partitions $\mathcal{P}(j)$ of $j$ For our largest $j=26$ this number is $\mathcal{P}(26)=2436$, which is still tractable for an exact diagonalization Without the decoupling of the entive Fock space into distinct layers, such large excitation energies would not be accessible numerically The layes approximation becomes more reasonable for larger $g$, because then $V \ll \Delta$ so that states fiom different layers may be regarded as uncoupled

The inverse participation ratio

$$
I=\sum_{m}|\langle\alpha \mid m\rangle|^{4}
$$

of the eigenstate $|\alpha\rangle$ of $H$ is the inverse of the number of eigenstates $|m\rangle$ of $H_{0}$ that have significant overlap with $|\alpha\rangle$ We calculate $I$ as a function of $g$ for diffeient layeıs $J$, corresponding to a mean excitation energy $\varepsilon=j \Delta$ The IPR fluctuates stiongly fiom state to state and for different realizations of the random matrix $H$ We calculate the averages $\bar{I}, \overline{1 / I}$, and $\overline{\ln I}$ where the overline "—" indicates an average both ovel the $\mathcal{P}(J)$ states $|\alpha\rangle$ in the $j$ th layer and over some $10^{3}$ ealizations of $H$ We first consider the logarithmic average $\overline{\ln I}$, for which the fluctuations are smallest

In Fig 1 we have plotted the numerical data for the $g$ dependence of $\overline{\ln I}$, for different values of $\varepsilon / \Delta$ In oider to compare with the analytical prediction of Ref [14], we have rescaled the variables such that Fig 1 becomes a plot of $-y^{-1} \overline{\ln I}$ versus $x$ The prediction is that, in the thermodynamic limit [18], the scaling function $F(x)=-y^{-1} \overline{\ln I}$ depends only on $x$

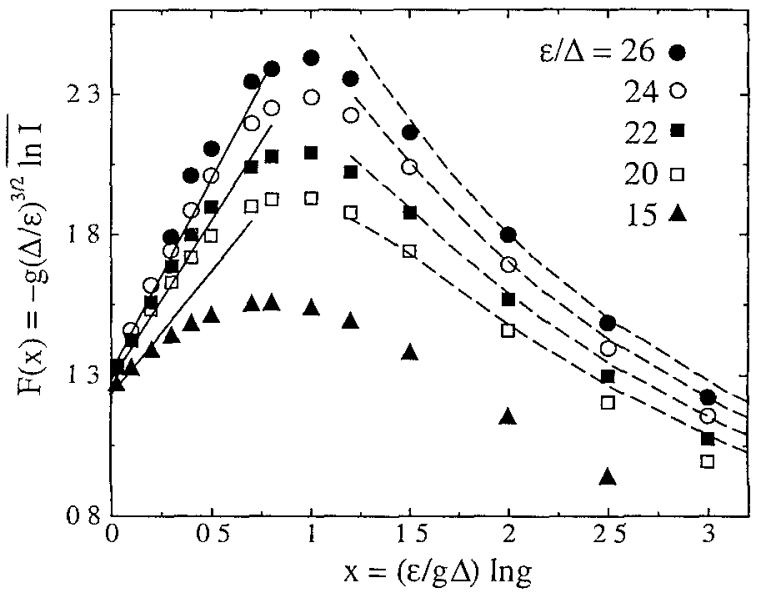

FIG 1 Average logarthm of the inverse patticipation ratio $I$ as a function of the dimensionless conductance $g$, in rescaled variables The diffeient sets of data points follow fiom the layel model for different excilation energies $j=\varepsilon / \Delta$ Statistical et ro1s are smaller than the size of the marke1s The straight solid lines are the analytical prediction (6) of the scaling theory, without any adjustable parameters (Only the lines for $\varepsilon / \Delta=15$, 20 , and 26 are shown for clanty) The dashed curves are the golden rule piediction (7), with a single adjustable parameter (the same for all curves, but the data for $\varepsilon / \Delta=15$ were left out of the fit)

for $x \leqslant 1$ This scalıng behavior cannot be checked directly because finite-size effects introduce an additional $\varepsilon$ dependence into the function $F(x)$ This is why we cannot directly test whether $x$ or $y$ is the correct scaling parameter Fortunately, it is possible to include finitesize effects in the scaling function and test the theory in this way

Applying the method of Ref [14] for the calculation of $\overline{\ln I}$ one finds that the function $F(x)$ in the thermodynamic limit has the Taylor series

$$
F(x)=-y^{-1} \overline{\ln I}=\sum_{n=0}^{\infty} c_{n} x^{n}
$$

with corrections of order $1 / \ln g$ All coefficients $c_{n}$ are positive The scaling behavior (3) is expected to be universal (valid for any model with random two-body interactions), but the coefficients $c_{n}$ are model specific The first two coefficients for the layer model are

$$
c_{0}=\frac{8(2-\sqrt{2})}{\sqrt{3 \pi}}=153, \quad c_{1}=\frac{81}{25} \sqrt{\frac{2}{\pi}} c_{0}=395
$$

In the thermodynamic limit the $n$-particle level spacing $\Delta_{n}$ equals $(\varepsilon / \Delta)^{-n+1 / 2} \Delta$ times a numerical coefficient of order unity Finite-size effects introduce an $\varepsilon$ dependence into this coefficient To quantify the finite-size effects, it is convenient to define the ratio 


$$
K_{n} \equiv\left(\frac{\Delta}{\varepsilon}\right)^{n-1 / 2} \frac{\Delta}{\Delta_{n}}
$$

The series expansion of $F(x)$ in terms of the $K_{n}$ 's is

$$
F(x)=4(\sqrt{2}-1) \sqrt{\pi} K_{2}+36(2-\sqrt{2}) K_{3} x+O\left(\lambda^{2}\right)
$$

Fo1 $\varepsilon / \Delta \rightarrow \infty$ we have $K_{2} \rightarrow(2 / \pi) \sqrt{2 / 3}=05198$, $K_{3} \rightarrow 6 \sqrt{6} / 25 \pi=01871$, and we recoves the theimodynamic limit (3) For the excitation eneigies $\varepsilon / \Delta=15$, $20,22,24$, and 26 of the simulation, after explicit calculation of $\Delta_{2}$ and $\Delta_{3}$, one finds $K_{2}=0419,0436,0439$, 0444 , and 0447 and $K_{3}=00414,00536,00577$, 00615 , and 00648 The resulting small- $x$ behavior of the scaling function is plotted in Fig 1 (solid lines) and agiees quite well with the numerical data

Analytically, the scaling function $F(x)$ is known only for $x \ll 1$ In the simulation, we observe a maximum of $-y^{-1} \overline{\ln I}$ at $x \simeq 1$ The maximum becomes more pronounced with increasing excitation energy We aigue that it is a signature of the tiansition to chaos, because beyond the maximum, for $x \geq 1$, the IPR is observed to follow the golden-1ule piediction (see discussion below)

$$
I_{\text {golden-rule }}=C\left[J^{5 / 4} \mathcal{P}(J)\right]^{-1} g^{2}
$$

This golden-rule piediction is shown dashed in Fig 1, with the coefficient $C \approx 051$ as the single fit parameter (The smallest $\varepsilon / \Delta=15$ was left out of the fit ) Note that $-y^{-1} \ln I_{\text {golden-rule }}$ has a maximum for an IPR of order unity, hence in the regime of localized states In contrast, the maximum in $-y^{-1} \overline{\ln I}$ occurs when the IPR 1 s $\ll 1$, hence in the regime of extended states We now discuss the small and large- $x$ regimes in some more detail

The large- $x$ legime is described by the golden rule $I_{\text {golden-1ule }}=\delta / \Gamma$, accoiding to which all basis states withın the decay width $\Gamma$ of a noninteracting state are equally mixed into the exact eigenstate This complete mixing amounts to fully developed chaos For oul model the level spacing of the many-particle states in the $j$ th layel is $\delta \sim J^{1 / 4} \Delta / \mathcal{P}(J)$ and the Bieit-Wigner width is $\Gamma \sim V^{2} / \Delta_{2} \sim j^{3 / 2} g^{-2} \Delta$, which leads to Eq (7) One notices in Fig 1 that for the laigest $x$ the data points fall somewhat below the golden-rule prediction This is due to the finite bandwidth of the layer model The IPR saturates at $3 / \mathcal{P}(J)$ [9] when the decay width $\Gamma$ becomes comparable to the bandwidth $j^{1 / 4} \Delta$ The corresponding upper bound on $x$ for the valıdity of the golden rule is $x \leqslant j^{3 / 8} \operatorname{lng}$ The finite bandwidth of the layer model becomes less significant for large $J$, which is why the agreement with the golden iule improves with increasing $J$
The small- $\lambda$ legime is described by the scaling function $F(x)$ The term of order $x^{n}$ in the Taylor senes (3) contains the $(n+1)$ th order effective inteiaction $V_{n+1}^{\text {eft }}$ between $n+2$ particles and holes A Fock state in the $j$ th layer contains about $\sqrt{j}$ excited paiticles and holes [19] Because this is a laige number for $y \gg 1$, the IPR factol izes into a product of independent contilbutions fiom $2,3,4$, inteiacting particles,

$$
\overline{\ln I} \sim \sum_{n=0}^{\infty} \overline{\left|V_{n+1}^{\mathrm{eff}}\right|} / \Delta_{n+2}
$$

A calculation of $\overline{\left|V_{n+1}^{\text {eff }}\right|}$ leads to Eq (3) The appearance of the modulus of the matrix element in Eq (8) is easily understood for the case of only two unperturbed manyparticle states interacting via the mati1x element $V^{\text {eff }}$ The IPR changes by or der unity if two Fock states come energetically within a separation $\left|V^{\text {eff }}\right|$ of each other The probability of such a neal degeneracy is small like $\left|V^{\text {eff }}\right| / \Delta$ (There is no level repulsion for the manyparticle solutions of the noninteracting Hamiltonian) Because toi weak interaction the IPR can change significantly but only with a small probability, the IPR fluctuates strongly Indeed, in our simulations much larger statistics was necessary in order to reach good accuracy in the small- $x$ regıme (The remainıng statıstical error in Fig 1 is smaller than the size of the markers )

In Fig 2 we compare the logaithmic average $\overline{\ln I}$ with the two other averages $\ln \bar{I}$ and $-\ln \overline{1 / I}$ Within the small$x$ regime of validity of Eq (3) the three averages are ielated by

$$
\overline{\ln I}=2(2-\sqrt{2}) \ln \bar{I}=-2(\sqrt{2}-1) \ln \overline{1 / I}
$$

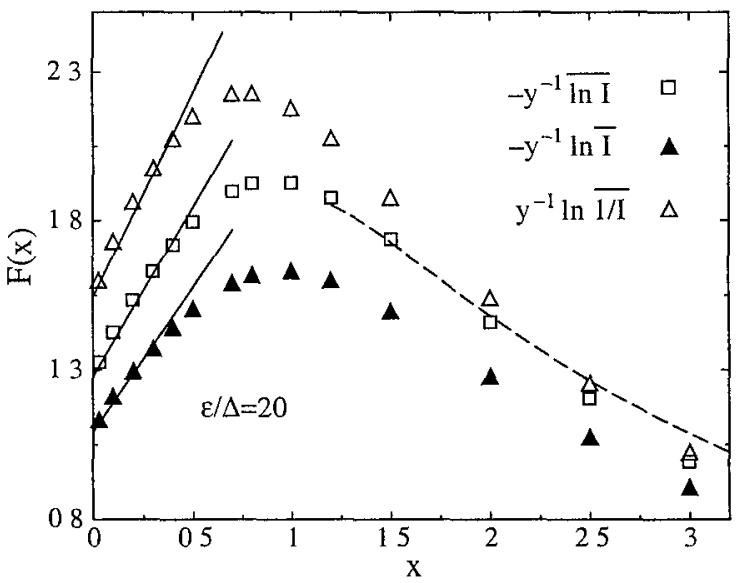

FIG 2 Averages $-\overline{\ln I},-\ln \bar{I}$, and $\ln \overline{1 / I}$ as a function of $g$, rescaled in the same way as in Fig 1 , for $\varepsilon / \Delta=20$ For small $x$, the thi ee averages follow the scaling theory (9) (solid lines) For large $x$ the averages $-\overline{\ln I}$ and $\ln \overline{1 / I}$ follow the golden rule (dashed line) 
These numerical coefficients do not depend on the number of particles involved in the interaction (cf the explicit calculation of $\bar{I}$ in Ref [14]) As one can see in Fig 2, for $\varepsilon / \Delta=20$, the relation (9) agrees well with the simulation In the chaotic legime, for latge $x, \mathrm{Eq}$ (9) is no longer valid The average $-\ln \overline{1 / 1}$, which is dominated by the majority of states having a large number of components, is close to $\overline{\ln I}$ at large $x$ The average $\ln \bar{I}$ is dom1nated by raie states with an anomalously small number of components and falls below the two other averages This indicates an asymmetic disti1bution of $\ln I$ in the chaotic legime for the layer model

So far we have addiessed only the question of the scalIng variable that goveins the transition to chaos What 1emains is the question How sharp is the transition? The singula1 threshold predicted in Ref [14] develops only in the thermodynamic limit and would be smoothed by finitesize effects in any simulation The corresponding nonanalyticity of $\overline{\ln I}$ is related to the high-order behavior of the series (3) Since our numerics allows us to distinguish only the first two coefficients $c_{0}$ and $c_{1}$, it leaves open the question about the nonanalyticity Still, even if the series (3) would be absolutely convergent, the resulting smooth function of the single variable $x$ could not descise the IPR for laige $x$ because it is incompatible with the golden iule $-y^{-1} \ln I_{\text {golden-rule }} \sim x^{-1} \ln g$ This different scaling behavioi for small and large values of $x$ suggests that the peak observed in Fig 2 would evolve into a singular threshold in the theimodynamic limit The only way to maintain a smooth ciossover would be to introduce a parametically large interpolating region between the two different scaling iegimes We cannot exclude this interpolating region on the basis of the numerical data, however, theoretically [14] there is no indication for such a region

In summary, by exact diagonalization of a model Hamıltonian we have presented evidence for an interactioninduced transition to chaos in a quantum dot Upon inclusion of finite-size effects, a good agreement is obtained with the scaling theory of Ref [14], supporting the assertion that $x=(\varepsilon / g \Delta) \ln g$ is the scaling parameter for the transition The different behavior of the scalıng function for small and large $x$ suggests that the transition would become a singular threshold in the thermodynamic limit

This work was supported by the Dutch Science Foundation NWO/FOM and by the TMR program of the European Commission The research of $\mathrm{P} G \mathrm{~S}$ was supported by RFBR, Giant No 98-02-17905 Discussions with J Tworzydło a1e gratefully acknowledged

-Present addiess Labolatone de Physique, Ecole Nolmale Superieure, 24 1ue Lhomond, 75231 Pars Cedex 05, Fiance

[1] E P Wigner, SIAM Rev 9, 1 (1967)

[2] O Bohıgas, M-J Grannonı, and C Schmıl, Phys Rev Lett 52, 1 (1984)

[3] T Guhı, A Muller-Groelıng, and H A Weıdenmulleı, Phys Rep 299, 189 (1998)

[4] Conelated Fermons and Tiansport m Mesoscopic Sys tems edited by Th Martın, G Montambaux, and J Trân Thanh Vân (Editıons Frontıè̀es, Gtf sut Yvette, 1996)

[5] B L Altshuler, Y Gefen, A Kamenev, and L S Levitov, Phys Rev Lett 78, 2803 (1997)

[6] P Jacquod and D L Shepelyansky, Phys Rev Lett 79, 1837 (1997)

[7] A D Mirlin and Y V Fyodorov, Phys Rev B 56, 13393 (1997)

[8] P G Silvestiov, Phys Rev Lett 79, 3994 (1997)

[9] C Mejia-Monasterio, J Richert, T Rupp, and H A We1denmuller, Phys Rev Lett 81, 5189 (1998)

[10] X Leyronas, J Tworzydło, and C W J Beenakket, Phys Rev Lett 82, 4894 (1999)

[11] V V Flambaum and F M Izrallev, Phys Rev E 56, 5144 (1997)

[12] B Georgeot and D L Shepelyansky, Phys Rev Lett 79, 4365 (1997)

[13] R Berkovits and Y Avisha1, Phys Rev Lett 80, 568 (1998)

[14] P G Silvestrov, Phys Rev E 58, 5629 (1998)

[15] Ya M Blanter, Phys Rev B 54, 12807 (1996)

[16] J B French and S S M Wong, Phys Lett B 33, 449 (1970), 35, 5 (1971)

[17] O Bohigas and J Flores, Phys Lett B 34, 261 (1971), 35, 383 (1971)

[18] By the thermodynamic limit we mean the limit of high excitation eneigy and large conductance, namely, $\varepsilon, g \rightarrow$ $\infty$ at fixed $x=(\varepsilon / \Delta)(\ln g / g)$

[19] The probability to find the single-particle level $\varepsilon$, (with excitation energy,$\Delta$ ) occupied in a Fock state is $p(\jmath)=$ $\left(e^{J / T}+1\right)^{-1}$ It has the form of a Fermi-Dirac distribution at an effective temperature $T=\sqrt{6 J} / \pi$ This formula, though formally valid only in the thermodynamic limit $T \gg 1$, describes well the average occupation number already at the values $T=35-4$ of our simulations 